\title{
STUDIES OF FRACTAL ASPECTS OF CEMENT
}

\author{
J.A. JANIK ${ }^{a}$, W. KURDoWSKI ${ }^{b}$, R. PoDSIADLY ${ }^{c}$ AND J. SAMSETH ${ }^{d}$ \\ ${ }^{a} \mathrm{H}$. Niewodniczański Institute of Nuclear Physics \\ Radzikowskiego 152, 31-342 Kraków, Poland \\ ${ }^{b}$ Mining Academy, Mickiewicza 30, 30-059 Kraków, Poland \\ ${ }^{c}$ Faculty of Chemistry of the Jagiellonian University \\ Ingardena 3, 30-060 Kraków, Poland \\ ${ }^{d}$ Institutt for energiteknikk, 2007 Kjeller, Norway
}

(Received May 22, 1996; in final form October 22, 1996)

\begin{abstract}
Small angle neutron scattering can be used to reveal the fractal nature of matter. The problems of fractal aspdcts of the structure of cement are related to those of fractal aspects of silica aerogels. Natural cement, when mixed with water, transforms after a series of chemical reactions (in a large part) into an amorphous phase called C-S-HI. It is believed that the cationic sublattice composed of $\mathrm{CaO}_{6}$ octahedrons constitutes the C-S-H structural basis, to which the $\mathrm{SiO}_{4}$ tetrahedral chains are attached. It is a layer structure in which the cation sublattice determines the way of $\mathrm{SiO}_{4}$ condensation. It is natural to expect that in this way one may obtain a really amorphous substance, since some tetrahedrons may be lacking, which leads to defects. We present here the results of a small angle neutron scattering and X-ray diffraction study of several samples with the pure C-S-H phase for which during the process of chemical synthesis various ratios of molar contents of $\mathrm{CaO}$ and $\mathrm{SiO}_{2}$ were used (various $\mathrm{C} / \mathrm{S}$ ratios). We obtained a gradual change of fractal characteristics with the change of $\mathrm{C} / \mathrm{S}$ ratio.
\end{abstract}

PACS numbers: $61.12 . \mathrm{Ex}, 82.70 . \mathrm{Gg}$

\section{Introduction}

The problems of fractal aspects of the structure of cement are related to those of fractal aspects of silica aerogels. The latter were subjected to various studies performed by the small angle neutron scattering (SANS) method, which provides not only the evidence, but also a quantitative description of the fractal structure [1-3]. Those studies analyzed the linear part of the dependence: logarithm of the scattered neutron intensity vs. logarithm of the neutron momentum transfer. The existence of such a linear part can be treated as a proof of a power law character of the substance density correlation function (pseudo Ornstein-Zernicke function) [1-3]:

$$
g(r) \sim r^{D-3} \exp (-r / \xi),
$$

where $\xi$ is the correlation length and $D$ is the Hausdorff dimension [4] of the 
analyzed sample $(0 \leq D \leq 3)$. Hence, one obtains a neutron scattering law (Ornstein-Zernicke law) $[1,2,5]$ :

$$
I(q) \sim q^{-D} \quad \text { or } \quad \log I(q) \sim-D \log q .
$$

The Hausdorff dimension $D$ is now a slope coefficient of the linear part of the $\log I$ vs. $\log q$ dependence (if it does exist, of course). If $D$ is smaller than 3 , it indicates the fractal behaviour of the sample and $D$ is called the fractal dimension [1-4]. In the papers concerning this problem, the fractal dimension $D=2.4$ was obtained for common silica aerogels. The linear part existed in a large range of momentum transfers $Q$ (from $c a .0 .005$ to $0.100 \AA^{-1}$ ).

There are cases when the dependence logarithm of scattered intensity vs. logarithm of momentum transfer has a slope which if treated in the same way would lead to a dimension larger than 3 , which is nonsense. Therefore, Porod has suggested that the slope arises from the surface of the scattering clusters in the sample, and introduced the following density correlation function [5]:

$$
g(r) \sim 4 \varphi(1-\varphi) r^{2-D_{\mathrm{a}}},
$$

where is the volume fraction of scattering clusters in the sample and $D_{\mathrm{s}}$ is the Hausdorff dimension of the cluster's surface $\left(2 \leq D_{\mathrm{s}} \leq 3\right)[2,3]$. It provides the following neutron scattering law [2]:

$$
I(q) \sim q^{-\left(6-D_{\mathrm{s}}\right)} \quad \text { or } \quad \log I(q) \sim-\left(6-D_{\mathrm{s}}\right) \log q .
$$

If $D_{\mathrm{s}}$ is greater than 2, it means a fractal behaviour of the cluster's surface and $D_{\mathrm{s}}$ is called the surface fractal dimension $[2,3]$.

An extension of these problems to natural cement may be summarized as follows $[6,7]$. The natural cement, when mixed with water, transforms after a series of hydratation and hydrolysis chemical reactions into a "cement paste". The cement paste is in a majority an amorphous system with only a little amount of crystalline phases. The amorphous phase is called C-S-H. It consists of the
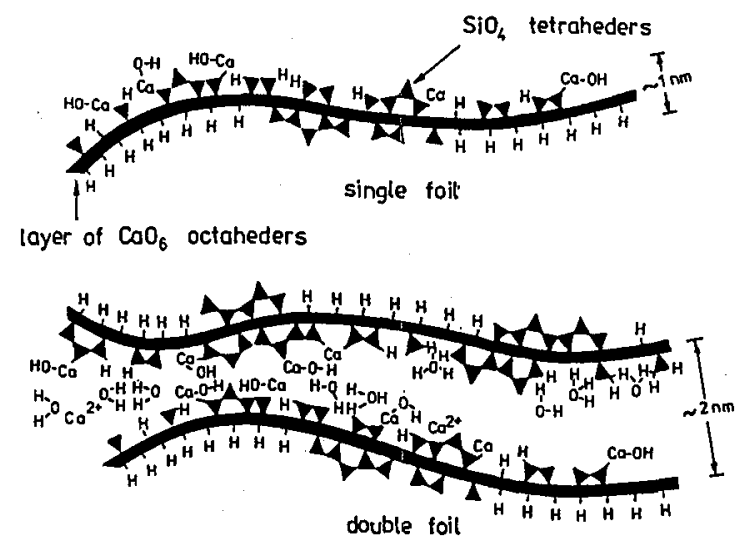

Fig. 1. Schematic picture of the structure of cement (after [7], courtesy of W. Kurdowski). 
tri- and di-calcium-silicate compounds with a different $\mathrm{Ca} / \mathrm{Si}(\mathrm{C} / \mathrm{S})$ molar ratios (e.g.: $\mathrm{Ca}_{2} \mathrm{SiO}_{4} \cdot n \mathrm{H}_{2} \mathrm{O}, \mathrm{Ca}_{3} \mathrm{Si}_{2} \mathrm{O}_{7} \cdot n \mathrm{H}_{2} \mathrm{O}, \mathrm{Ca}_{3} \mathrm{Si}_{3} \mathrm{O}_{9} \cdot n \mathrm{H}_{2} \mathrm{O}$, and so on). The C-S-H phase fully determines behaviours of the paste [6]. It is believed that the cationic sublattice, which is composed of the $\mathrm{CaO}_{6}$ octahedrons, constitutes its structural basis, to which the $\mathrm{SiO}_{4}$ tetrahedral chains are attached. This is a layer structure in which the cation sublattice determines the way of $\mathrm{SiO}_{4}$ condensation. It is natural to expect that in this way one may obtain a really amorphous substance, since some tetrahedrons may be lacking, which leads to defects. This rather complicated situation is shown in Fig. 1 [7].

Taking the mentioned cement features into account, in this paper we present results of a X-ray and a SANS study of several samples, consisting of the pure C-S-H phase which we treat as a model of the real cement paste. Various ratios of molar contents of $\mathrm{CaO}$ and $\mathrm{SiO}_{2}$ were used (various $\mathrm{C} / \mathrm{S}$ ratios) during the process of chemical synthesis. As it is shown in the following sections, we obtained a gradual change of fractal characteristics with the change of $\mathrm{C} / \mathrm{S}$ ratios.

\section{Samples and their X-ray diffraction testing}

To accelerate the C-S-H phase preparation an amorphous $\mathrm{SiO}_{2}$ was used (the Aerosil product of the Degussa Company). The specific surface of that product was about $200 \mathrm{~m}^{2} / \mathrm{g}$. The C-S-H phase was obtained from an aqueous suspension

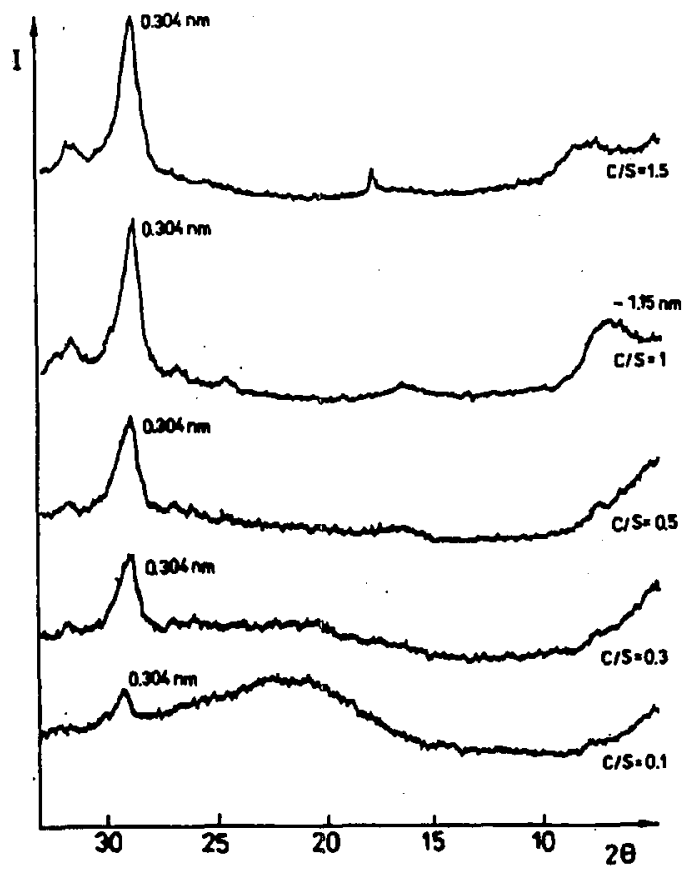

Fig. 2. X-ray diffraction patterns for samples with various $\mathrm{C} / \mathrm{S}$ ratios. The spectra are shifted along the " $I$ "-axis to clarify the picture. 
of $\mathrm{SiO}_{2}$ and $\mathrm{Ca}(\mathrm{OH})_{2}$ by shaking in the course of many weeks (for the crystalline $\mathrm{SiO}_{2}$ this process needs much longer time). Various $\mathrm{C} / \mathrm{S}$ ratios were obtained by selecting proper molar quantities of the reacting substances. The suspension was carefully protected from the atmospheric $\mathrm{CO}_{2}$ during the process of shaking. Five samples were prepared in this way - with $\mathrm{C} / \mathrm{S}=0.1,0.3,0.5,1.0$ and 1.5.

The X-ray diffraction patterns (Fig. 2) show clearly that the sample with the lowest $\mathrm{C} / \mathrm{S}$ ratio $(0.1)$ is amorphous, as evidenced by the broad band in the central part of the pattern. A trace of the broad band can be seen also for $\mathrm{C} / \mathrm{S}=0.3$. Further patterns show an increasing crystallinity. It is evidenced by two reflexes mainly, one at $c a .0 .3 \mathrm{~nm}$ and another at $c a .1 .1 \mathrm{~nm}$, both characteristic of the tobermorite structure.

Basing on X-ray diffraction patterns solely, we can form the following picture: at $\mathrm{C} / \mathrm{S}=\mathbf{0 . 1}$ an amorphous silica gel, which is a porous substance, will be dominant. Then gradually the C-S-H substance is formed. It is also amorphous but with crystallites resulting from the ordering by attachment of $\mathrm{SiO}_{4}$ chains to $\mathrm{CaO}_{6}$ layers as explained in Sec. 1 . One has to notice that the $\mathrm{X}$-rays give evidence of the increasing amount of crystallites but the X-rays are not so good as means of detecting the disorder which comes from increasing number of defects due to lack of $\mathrm{SiO}_{4}$ tetrahedrons. From this point of view the SANS method seems to be complementary to the X-ray diffraction.

\section{SANS results}

Figure 3 presents the SANS results for the five samples. We believe that the slope is dominated by the surface fractals. If so, one should try to obtain the surface fractal dimension by subtracting 6 from the negative slope (formula (4) in Sec. 1). Figure 4 and Table present the so obtained $D_{\mathrm{s}}$ values for our samples. The surface fractal dimension $D_{\mathrm{s}}$ as obtained from the slope for small $\mathrm{C} / \mathrm{S}$ is not far from $2, D_{\mathrm{s}}=2.2$, which is not very different from what was obtained in [1], where $D_{\mathrm{s}}=2.0$. Perhaps this means that the walls of the pores are rather smooth. When

TABLE

Surface fractal dimension $\left(D_{s}\right)$ as a function of $\mathrm{CaO} / \mathrm{SiO}_{2}$ ratio.

\begin{tabular}{c|c}
\hline \hline C/S ratio & $D_{\mathrm{s}}$ \\
\hline 0.1 & $2.16 \pm 0.02$ \\
0.3 & $2.20 \pm 0.02$ \\
0.5 & $2.34 \pm 0.03$ \\
1.0 & $2.39 \pm 0.03$ \\
1.5 & $2.44 \pm 0.02$
\end{tabular}

$\mathrm{C} / \mathrm{S}$ ratio increases the substance becomes gradually C-S-H, containing $\mathrm{CaO}_{6}$ foils to which the $\mathrm{SiO}_{4}$ tetrahedrons become attached, the walls of the pores become less and less smooth which should lead to an increase in $D_{\mathrm{s}}$. And that is what we indeed observe. 


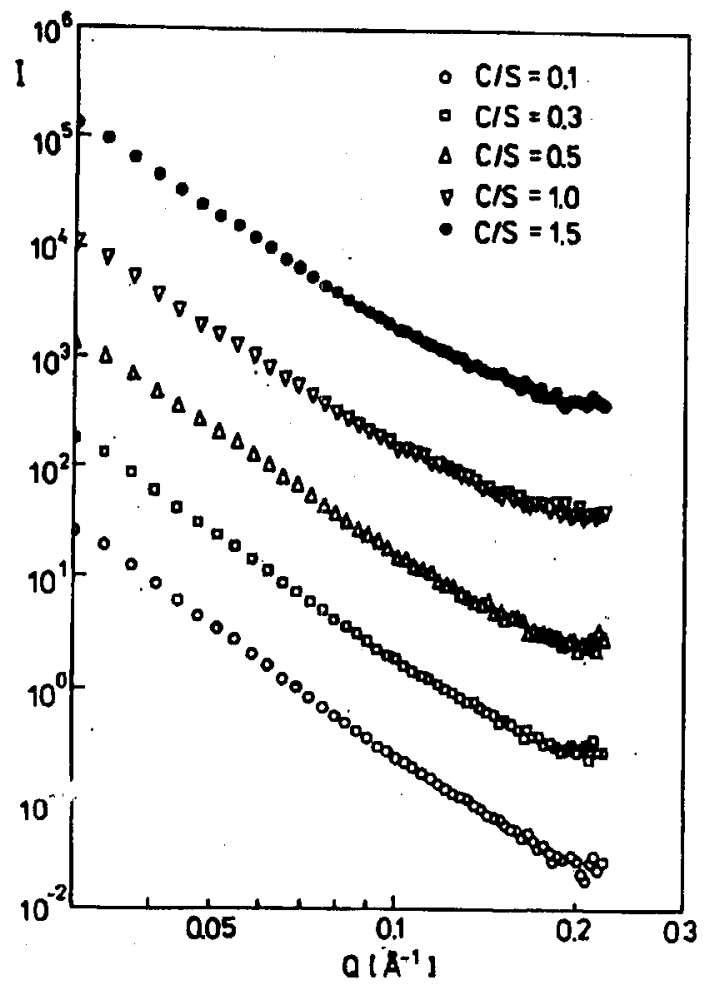

Fig. 3. SANS diffraction patterns for samples with various $\mathrm{C} / \mathrm{S}$ ratios. The spectra are shifted along the "I"-axis to clarify the picture.

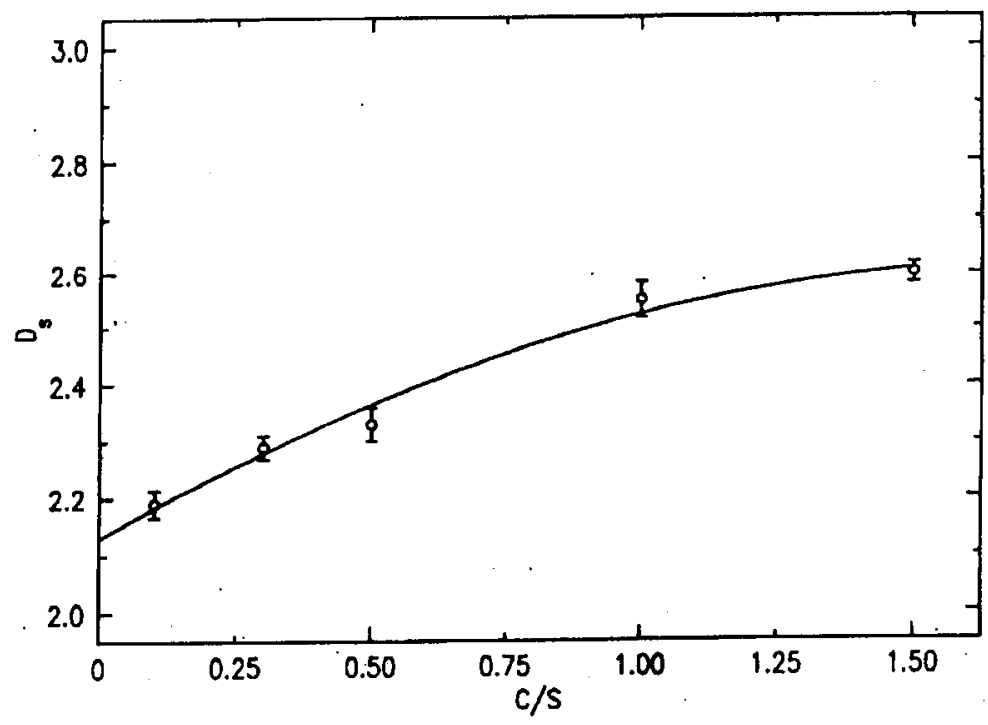

Fig. 4. SANS results for samples with various $\mathrm{C} / \mathrm{S}$ ratios. 


\section{Conclusions}

Five samples of C-S-H gels were studied, with attention paid to their increasing $\mathrm{CaO} / \mathrm{SiO}_{2}$ molar ratio from 0.1 to 1.5 . The $\mathrm{X}$-ray diffraction gave evidence of an amorphous structure at low $\mathrm{C} / \mathrm{S}$ values and of an increasing crystallinity for the C-S-H phase at higher C/S. The SANS study gave evidence of an increasing fractal character of the surface of the pores which accompanies the process of crystallites formation as evidenced by X-rays. The walls of the pores become surface fractals.

\section{References}

[1] R. Vacher, T. Woigner, J. Pelous, E. Courtens, Phys. Rev. B 37, 6500 (1988).

[2] E. Courtens, R. Vacher, Proc. R. Soc. Lond. A 423, 55 (1989).

[3] J.K. Kjems, D. Posselt, in: Random Fluctuations and Pattern Growth: Experiments and Models, Eds. H.E. Stanley, N. Ostrovsky, Kluwer Academic Publishers, Dordrecht 1988, p. 7.

[4] G. Edgar, Measure, Topology and Fractal Geometry, Springer Verlag, New York 1990.

[5] G. Porod, in: Small Angle X-ray Scaltering, Eds. O. Glater, O. Kratky, Academic Press, London 1982, Ch. 2.

[6] H.F.W. Taylor, The Chemistry of Cements, Academic Press, London 1964.

[7] W. Kurdowski, Cement Chemistry, PWN, Warszawa 1991 (in Polish). 